\title{
Work Life Balance of Airhostess at Airlines Sector
}

\author{
Asima Aziz ${ }^{1}$, Dr. Y. Vinodhini ${ }^{2}$ \\ \{ruhiasima@gmail.com ${ }^{1}$, adithyasiddartha13@gmail.com² ${ }^{2}$, \\ Associate Professor, Dept of management studies \\ Deccan School of Management ${ }^{1}$, Professor, Dept of management studies \\ Anwarul Uloom College of Business Management, Hyderabad
}

\begin{abstract}
Work-life balance is particularly crucial when an organization must handle highly technical professionals because their strong dedication and loyalty are required for the organization's success. Aviation is a strategically important sector of any economy. People working in the aviation industry, particularly the flight attendants/ Air hostess. It is an interesting place to be for people who love to travel, see new sights and experience the ultimate adrenaline rush. Many issues throw into Air hostess not understanding sufficient work life balance including uneven and extended working hours, travelling, spending a lot of time absent from home and the inflexibility of time schedules. This paper empirically analyzes the factors influencing social and emotional factors of airhostess in aviation sector..
\end{abstract}

Keywords: Work Life Balance, organization, commitment, women, airhostess, factors.

\section{Introduction}

Work life balance usually pleased with their functioning arrangements (Eikhof D. R., 2007). Work/life balance has become a principal issue in the workplace. Marital status, working hours, necessity of flexibility, added working hours and over time distort the work life balance (Fleetwood S., 2007). Basically the main purpose of work life balance is to have a growth in both in professional and personal growth or satisfaction (Yadav R.K., \& Dabhade N., 2014).

\section{Air Hostess}

An air hostess, also called as a flight attendant or stewardess, are employees of airline company (Williams C., 2003). They are authorized by federal law to observe public security and guarantee the fulfillment of customers with airline safety systems. They hold the in-flight requirements of clients, administer first aid to those who become unwell, and provide food and beverages (Barry K., 2007). They typically work long hours during which they must do diverse responsibilities.

\section{Job satisfaction}

Job satisfaction mostly appears at the level to which employees have optimistic or unenthusiastic manner towards their employment (Ahmad M. B., Wasay E., \& Jhandir S. U., 2012). An attitude is an individual employee's feeling (satisfaction, indifference or dissatisfaction) towards a precise condition, object or person. Job satisfaction is the net effect of the superior or poor approach held by an individual employee at a given phase of time 
(Maeran R., Pitarelli F., \& Cangiano F., 2013). It is subject to swings from one extreme to the other but typically reverts to a quite even stage that can be good or poor.

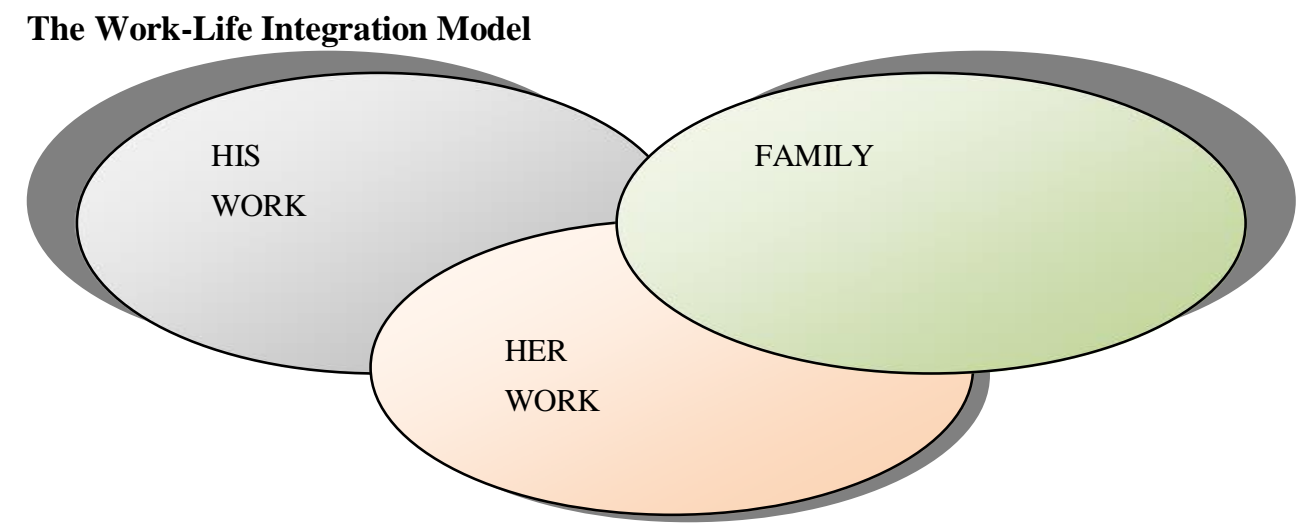

Source: (Barnett R. , A New Work-Life Model for the Twenty-First Century, 1999)

The above model expresses an understanding for employees and their lives outside of work. This model takes into consideration the needs of its employee's partners or spouses (Fan W., et al., 2015). This is to ensure employers can retain the best employees as well as ensuring optimum working productivity from its employees. Although this model has come about no new formal work-life policies have resulted which recognize men and women as multidimensional beings who participate in several roles through their lives (Barnett R., 1999).

\section{Review of Literature}

Work-life harmony is a crucial principle. All has to strike a balance between their work and family lives in order to live a happy life. This is why employers now choose businesses that have exclusive and appealing work-life balance plans. As a result, many companies implemented Work-Life Balance practices to recruit more workers and minimize work-life tension among current staff in order to improve organizational success (Beauregard T. A., \& Henry L. C., 2009).

\section{Work Life balance}

1. Lockwood (2003) defined work- life balance is a method of balancing work and personal obligations. Senior management would promote work-life activities. An organizational culture that allows workers to look at business in a completely different manner and respects and embraces employees as people with interests outside of the workplace is beneficial for work-life opportunities in the workplace. Employee motivation and morale was increased by work-life balance systems.

\section{Work life balance at Aviation sector}


1. Liang and Hsieh (2007), the airline industry's turnover rate remains high due to the difficulty of the job and the delay challenge to strike a balance between home and work life.

2. Chung and Chung (2009, p. 217) state "flight attendants have demonstrated abnormally high degrees of fatigue." Therefore, health remains an important part of a flight attendants ability to do his or her job effectively

\section{Social and emotional factors of work life balance}

3. Karasek and Theorell, (1990, p. 6)Numerous terms are used to describe social support; the most common of which is the beneficial social interactions between managers and colleagues in the organization

4. Brown, Prashantham and Abbott (2003) contend about the burnout of employees that social support provided from managers and colleagues have more influence on buffering employees' burnout.

\section{Objectives of the study:}

1. To study the conceptual framework of work life balance

2. To analyze social and emotional factors of airhostess in aviation sector.

\section{Hypothesis}

$\mathbf{H}_{\mathbf{0}}$ : There is no significant impact of work life balance aspects and Work life balance at aviation sector

Ho: Age of respondents has no significant impact on work life balance aspects

\section{Research Methodology}

Type of study: Descriptive

Primary Data: Primary data has been collected with data collection instrument (Questionnaire) administered to the respondents

Sources of data: Secondary data has been sourced for the present study surf engines, journals, and magazines.

\section{Limitations of the study}

- Small sample has been selected for the study

- The information gathered may be biased

- Time is one of the limiting factors

\section{Data analysis and interpretation}

Table-1: Age

\begin{tabular}{|l|l|l|}
\hline Age & Respondents & $\%$ \\
\hline $17-25 y e a r s$ & 102 & 68 \\
\hline $25-35$ & 48 & 32 \\
\hline
\end{tabular}




\begin{tabular}{|l|l|l|}
\hline Total & 150 & $100 \%$ \\
\hline
\end{tabular}

\section{Source: Based on Primary Data}

Analysis: From the above table it is clear that 68 percent respondents are in the age group of 17-25 years and remaining 32 percent respondents are in the age group of 25-35 years.

Table-2: Marital Status

\begin{tabular}{|l|l|l|}
\hline Marital Status & Respondents & \% \\
\hline Married & 25 & 17 \\
\hline Unmarried & 125 & $\mathbf{8 3}$ \\
\hline Total & 150 & 100 \\
\hline
\end{tabular}

Source: Based on Primary Data

Analysis: It is depicted from the table above pertaining to marital status of sample respondents, that out of 150 sample size of respondents (airhostess) 83 percent respondents are unmarried and remaining 17 percent respondents are married.

Table-3: Qualities of airhostess

\begin{tabular}{|l|l|l|l|l|l|l|}
\hline $\begin{array}{l}\text { Qualities of } \\
\text { airhostess }\end{array}$ & $\begin{array}{l}\text { Highly } \\
\text { importa } \\
\text { nt }\end{array}$ & $\begin{array}{l}\text { Importan } \\
\text { t }\end{array}$ & $\begin{array}{l}\text { Neither/ } \\
\text { nor }\end{array}$ & $\begin{array}{l}\text { Not } \\
\text { important }\end{array}$ & $\begin{array}{l}\text { Highly } \\
\text { unimport } \\
\text { ant }\end{array}$ & Total \\
\hline Multi-tasking & $\mathbf{5}$ & $\mathbf{8}$ & $\mathbf{2}$ & $\mathbf{2}$ & $\mathbf{1}$ & $\mathbf{1 8}$ \\
\hline empathy & $\mathbf{4}$ & $\mathbf{1 1}$ & $\mathbf{1}$ & $\mathbf{1}$ & $\mathbf{1}$ & $\mathbf{1 8}$ \\
\hline Situation awareness & $\mathbf{4}$ & $\mathbf{4}$ & $\mathbf{2}$ & $\mathbf{1}$ & $\mathbf{1}$ & $\mathbf{1 2}$ \\
\hline learning potential & $\mathbf{3}$ & $\mathbf{8}$ & $\mathbf{2}$ & $\mathbf{1}$ & $\mathbf{1}$ & $\mathbf{1 5}$ \\
\hline $\begin{array}{l}\text { Tolerant and } \\
\text { flexible }\end{array}$ & $\mathbf{3}$ & $\mathbf{5}$ & $\mathbf{2}$ & $\mathbf{1}$ & $\mathbf{1}$ & $\mathbf{1 2}$ \\
\hline Patience & $\mathbf{3}$ & $\mathbf{8}$ & $\mathbf{1}$ & $\mathbf{1}$ & $\mathbf{1}$ & $\mathbf{1 4}$ \\
\hline discipline and & $\mathbf{3}$ & $\mathbf{6}$ & $\mathbf{1}$ & $\mathbf{1}$ & $\mathbf{1}$ & $\mathbf{1 2}$ \\
\hline $\begin{array}{l}\text { style } \\
\text { glamorous }\end{array}$ & $\mathbf{1 2}$ & $\mathbf{2}$ & $\mathbf{1}$ & $\mathbf{1}$ & $\mathbf{1 9}$ \\
\hline kindness & $\mathbf{4}$ & $\mathbf{8}$ & $\mathbf{2}$ & $\mathbf{1}$ & $\mathbf{1}$ & $\mathbf{1 6}$ \\
\hline Work in team & $\mathbf{6}$ & $\mathbf{4}$ & $\mathbf{2}$ & $\mathbf{1}$ & $\mathbf{1}$ & $\mathbf{1 4}$ \\
\hline Total & $\mathbf{3 8}$ & $\mathbf{7 4}$ & $\mathbf{1 7}$ & $\mathbf{1 1}$ & & $\mathbf{1 0}$ \\
\hline
\end{tabular}

Source: Based on Primary Data

Analysis: From the above table it is found that with regards to the qualities of airhostess on Multi tasking 5 respondents mentioned as Highly important 8 respondents mentioned as important, 2 respondents mentioned as neither or nor, 2 respondents mentioned as unimportant and only 1 respondent mentioned as highly unimportant, with regards to empathy, 4 respondents mentioned as Highly important 11 respondents mentioned as important, 1 
respondent mentioned as neither or nor, 1 respondent mentioned as unimportant and 1 respondent mentioned as highly unimportant, with regards to the qualities of airhostess on Situation awareness 4 respondents mentioned as Highly important 4 respondents mentioned as important, 2 respondents mentioned as neither or nor, 1 respondent mentioned as unimportant and 1 respondent mentioned as highly unimportant, with regards learning potential, 3 respondents mentioned as Highly important 8 respondents mentioned as important, 2 respondents mentioned as neither or nor, 1 respondent mentioned as unimportant and 1 respondent mentioned as highly unimportant. with regards to Tolerant and flexible, 3 respondents mentioned as Highly important 5 respondents mentioned as important, 2 respondents mentioned as neither or nor, 1 respondent mentioned as unimportant and 1 respondent mentioned as highly unimportant, with regards to Patience level of respondents/airhostess 3 respondents mentioned as Highly important 8 respondents mentioned as important, 1 respondent mentioned as neither or nor, 1 respondent mentioned as unimportant and 1 respondent mentioned as highly unimportant, with regards to Discipline, 3 respondents mentioned as Highly important 6 respondents mentioned as important, 1 respondent mentioned as neither or nor, 1 respondent mentioned as unimportant and 1 respondent mentioned as highly unimportant, with regards to style and Glamour, 3 respondents mentioned as Highly important 12 respondents mentioned as important, 2 respondents mentioned as neither or nor, 1 respondent mentioned as unimportant and 1 respondent mentioned as highly unimportant, with regards to kindness, 4 respondents mentioned as Highly important 8 respondents mentioned as important, 2 respondents mentioned as neither or nor, 1 respondent mentioned as unimportant, 1 respondent mentioned as highly unimportant, and with regards to work in team 6 respondents mentioned as Highly important 4 respondents mentioned as important, 2 respondents mentioned as neither or nor, 1 respondents mentioned as unimportant 1 respondent mentioned as highly unimportant

Table-3: Work life balance at aviation sector

\begin{tabular}{|l|l|l|l|l|l|l|}
\hline & $\begin{array}{l}\text { Strongly } \\
\text { Agree }\end{array}$ & Agree & $\begin{array}{l}\text { Neither/no } \\
\text { r }\end{array}$ & Disagree & $\begin{array}{l}\text { Strongly } \\
\text { Disagree }\end{array}$ & total \\
\hline Very good experience & $\mathbf{5}$ & $\mathbf{8}$ & $\mathbf{6}$ & $\mathbf{1}$ & $\mathbf{1}$ & $\mathbf{2 1}$ \\
\hline $\begin{array}{l}\text { Multi cultural working } \\
\text { environment }\end{array}$ & $\mathbf{5}$ & $\mathbf{1 2}$ & $\mathbf{4}$ & $\mathbf{2}$ & $\mathbf{2}$ & $\mathbf{2 5}$ \\
\hline $\begin{array}{l}\text { Every day brings new } \\
\text { challenges }\end{array}$ & $\mathbf{3}$ & $\mathbf{3}$ & $\mathbf{2}$ & $\mathbf{2}$ & $\mathbf{2}$ & $\mathbf{1 2}$ \\
\hline Physically challenging & $\mathbf{1}$ & $\mathbf{2}$ & $\mathbf{2}$ & $\mathbf{1}$ & $\mathbf{2}$ & $\mathbf{8}$ \\
\hline Away from home & $\mathbf{9}$ & $\mathbf{3}$ & $\mathbf{1}$ & $\mathbf{1}$ & $\mathbf{1}$ & $\mathbf{1 5}$ \\
\hline Long working hours & $\mathbf{3}$ & $\mathbf{8}$ & $\mathbf{2}$ & $\mathbf{1}$ & $\mathbf{1}$ & $\mathbf{1 5}$ \\
\hline Lonely feeling & $\mathbf{2}$ & $\mathbf{3}$ & $\mathbf{1}$ & $\mathbf{2}$ & $\mathbf{2}$ & $\mathbf{1 0}$ \\
\hline Fatigue & $\mathbf{3}$ & $\mathbf{3}$ & $\mathbf{4}$ & $\mathbf{1}$ & $\mathbf{1}$ & $\mathbf{1 2}$ \\
\hline Constant progress and training & $\mathbf{7}$ & $\mathbf{1 0}$ & $\mathbf{3}$ & $\mathbf{1}$ & $\mathbf{1}$ & $\mathbf{2 2}$ \\
\hline Job security & $\mathbf{1}$ & $\mathbf{2}$ & $\mathbf{1}$ & $\mathbf{3}$ & $\mathbf{3}$ & $\mathbf{1 0}$ \\
\hline Total & $\mathbf{3 9}$ & $\mathbf{5 4}$ & $\mathbf{2 6}$ & $\mathbf{1 5}$ & $\mathbf{1 6}$ & $\mathbf{1 5 0}$ \\
\hline
\end{tabular}

Source: Based on Primary Data 
Analysis: Out of total 150 respondents 21 respondents said very good experience, 25 respondents mentioned as multicultural working environment, 12 respondents every day brings new challenges, 8 respondents physically challenging, 15 respondents away from home, 15 respondents said long working hours, 10 respondents mentioned as lonely feeling, 12 respondents mentioned as fatigue, 22 respondents mentioned as constant progress and training and remaining 10 respondents mentioned as job security.

Table-5: Work life balance aspects

\begin{tabular}{|l|l|l|l|l|l|}
\hline & $\begin{array}{l}\text { Strongly } \\
\text { Agree }\end{array}$ & Agree & $\begin{array}{l}\text { Neither/n } \\
\text { or }\end{array}$ & Disagree & Total \\
\hline Commitment fair & $\mathbf{8}$ & $\mathbf{8}$ & $\mathbf{2}$ & $\mathbf{1}$ & $\mathbf{2 2}$ \\
\hline $\begin{array}{l}\text { Adequate and } \\
\text { compensation }\end{array}$ & $\mathbf{5}$ & $\mathbf{1}$ & $\mathbf{1}$ & $\mathbf{1 5}$ \\
\hline Job satisfaction & $\mathbf{6}$ & $\mathbf{1 1}$ & $\mathbf{6}$ & $\mathbf{2}$ & $\mathbf{2 5}$ \\
\hline Training and development & $\mathbf{4}$ & $\mathbf{1 2}$ & $\mathbf{2}$ & $\mathbf{1}$ & $\mathbf{1 9}$ \\
\hline $\begin{array}{l}\text { Safe and healthy working } \\
\text { conditions }\end{array}$ & $\mathbf{3}$ & $\mathbf{1 4}$ & $\mathbf{2}$ & $\mathbf{2}$ & $\mathbf{2 1}$ \\
\hline Skill utilization and growth & $\mathbf{5}$ & $\mathbf{9}$ & $\mathbf{3}$ & $\mathbf{2}$ & $\mathbf{1 9}$ \\
\hline Social Integration & $\mathbf{1 2}$ & $\mathbf{5}$ & $\mathbf{2}$ & $\mathbf{1}$ & $\mathbf{2 0}$ \\
\hline Work environment & $\mathbf{6}$ & $\mathbf{4}$ & $\mathbf{3}$ & $\mathbf{2}$ & $\mathbf{1 5}$ \\
\hline Total & $\mathbf{4 6}$ & $\mathbf{7 1}$ & $\mathbf{2 1}$ & $\mathbf{1 2}$ & $\mathbf{1 5 0}$ \\
\hline
\end{tabular}

Source: Based on Primary Data

Analysis: Out of total 150 respondents 22 respondents mentioned as commitment, out of this 8 respondents mentioned as strongly agree 11 respondents as agree, 2 respondents mentioned as neither/nor, and only 1 respondent mentioned as disagree with regards to adequate and fair compensation(15) 8 respondents mentioned as strongly agree, 5 respondents mentioned as agree, 1 respondent mentioned as neither or nor and 1 respondent mentioned as disagree, with regards job satisfaction 6 respondents mentioned as strongly, 11 respondents mentioned as agree, 6 respondents mentioned as neither or nor, 2 respondents mentioned as disagree. with regards to training and development(19) 4 respondents mentioned as strongly agree, 12 respondents mentioned as agree, 2 respondents mentioned as neither or nor and 1 respondent mentioned as disagree, with regards to Safe and healthy working conditions(21) respondents 3 respondents mentioned as strongly agree, 14 respondents mentioned as agree, 2 respondents mentioned as neither or nor and 2 respondent mentioned as disagree, with regards to skill utilization and growth(19) 5 respondents mentioned as strongly agree, 9 respondents mentioned as agree, 3 respondents mentioned as neither or nor and 2 respondents mentioned as disagree, with regards to social integration(20 respondents), 12 respondents mentioned as strongly agree, 5 respondents mentioned as agree, 2 respondents mentioned as neither or nor and 1 respondent mentioned as disagree, with regards to work environment(15 respondents) 6 respondents mentioned as strongly agree, 4 respondents mentioned as agree, 3 respondents mentioned as neither or nor and 2 respondents mentioned as disagree

Table: 6 Crosstab of Work life balance at aviation sector and work life balance aspects 


\begin{tabular}{|l|l|l|l|l|l|l|l|l|l|}
\hline $\begin{array}{l}\text { Work life } \\
\text { balance at } \\
\text { aviation } \\
\text { sector/work } \\
\text { life balance } \\
\text { aspects }\end{array}$ & $\begin{array}{l}\text { Com } \\
\text { ent }\end{array}$ & $\begin{array}{l}\text { Adequate } \\
\text { and fair } \\
\text { compensatio } \\
\text { n }\end{array}$ & $\begin{array}{l}\text { Job } \\
\text { satisfactio } \\
\text { n }\end{array}$ & $\begin{array}{l}\text { Training } \\
\text { and } \\
\text { develop } \\
\text { ment }\end{array}$ & $\begin{array}{l}\text { Safe } \\
\text { and } \\
\text { healthy } \\
\text { workin } \\
\text { g } \\
\text { conditi } \\
\text { ons }\end{array}$ & $\begin{array}{l}\text { Skill } \\
\text { utiliza } \\
\text { tion } \\
\text { and } \\
\text { growt } \\
\text { hol }\end{array}$ & $\begin{array}{l}\text { Social } \\
\text { Integr } \\
\text { ation }\end{array}$ & $\begin{array}{l}\text { Work } \\
\text { envir } \\
\text { onme } \\
\text { nt }\end{array}$ & Total \\
\hline $\begin{array}{l}\text { Very good } \\
\text { experience }\end{array}$ & $\mathbf{2}$ & $\mathbf{3}$ & $\mathbf{2}$ & $\mathbf{3}$ & $\mathbf{2}$ & $\mathbf{3}$ & $\mathbf{3}$ & $\mathbf{3}$ & $\mathbf{2 1}$ \\
\hline $\begin{array}{l}\text { Multi cultural } \\
\text { working } \\
\text { environment }\end{array}$ & $\mathbf{4}$ & $\mathbf{2}$ & $\mathbf{3}$ & $\mathbf{5}$ & $\mathbf{3}$ & $\mathbf{3}$ & $\mathbf{3}$ & $\mathbf{2}$ & $\mathbf{2 5}$ \\
\hline $\begin{array}{l}\text { Every day } \\
\text { brings new } \\
\text { challenges }\end{array}$ & $\mathbf{1}$ & $\mathbf{2}$ & $\mathbf{2}$ & $\mathbf{1}$ & $\mathbf{2}$ & $\mathbf{1}$ & $\mathbf{1}$ & $\mathbf{2}$ & $\mathbf{1 2}$ \\
\hline $\begin{array}{l}\text { Physically } \\
\text { challenging }\end{array}$ & $\mathbf{1}$ & $\mathbf{1}$ & $\mathbf{1}$ & $\mathbf{1}$ & $\mathbf{1}$ & $\mathbf{1}$ & $\mathbf{1}$ & $\mathbf{1}$ & $\mathbf{8}$ \\
\hline $\begin{array}{l}\text { Away from } \\
\text { home }\end{array}$ & $\mathbf{1}$ & $\mathbf{2}$ & $\mathbf{2}$ & $\mathbf{2}$ & $\mathbf{1}$ & $\mathbf{3}$ & $\mathbf{2}$ & $\mathbf{2}$ & $\mathbf{1 5}$ \\
\hline $\begin{array}{l}\text { Long working } \\
\text { hours }\end{array}$ & $\mathbf{2}$ & $\mathbf{1}$ & $\mathbf{2}$ & $\mathbf{2}$ & $\mathbf{2}$ & $\mathbf{2}$ & $\mathbf{2}$ & $\mathbf{1}$ & $\mathbf{1 5}$ \\
\hline Lonely feeling & $\mathbf{2}$ & $\mathbf{1}$ & $\mathbf{1}$ & $\mathbf{2}$ & $\mathbf{1}$ & $\mathbf{1}$ & $\mathbf{1}$ & $\mathbf{1}$ & $\mathbf{1 0}$ \\
\hline Fatigue & $\mathbf{1}$ & $\mathbf{2}$ & $\mathbf{2}$ & $\mathbf{1}$ & $\mathbf{2}$ & $\mathbf{1}$ & $\mathbf{1}$ & $\mathbf{2}$ & $\mathbf{1 2}$ \\
\hline $\begin{array}{l}\text { Constant } \\
\text { progress } \\
\text { and training }\end{array}$ & $\mathbf{2}$ & $\mathbf{3}$ & $\mathbf{3}$ & $\mathbf{3}$ & $\mathbf{4}$ & $\mathbf{2}$ & $\mathbf{2}$ & $\mathbf{3}$ & $\mathbf{2 2}$ \\
\hline Job security & $\mathbf{1}$ & $\mathbf{1}$ & $\mathbf{1}$ & $\mathbf{2}$ & $\mathbf{2}$ & $\mathbf{1}$ & $\mathbf{1}$ & $\mathbf{1}$ & $\mathbf{1 0}$ \\
\hline Total & $\mathbf{2 2}$ & $\mathbf{1 5}$ & $\mathbf{2 5}$ & $\mathbf{1 9}$ & $\mathbf{2 1}$ & $\mathbf{1 9}$ & $\mathbf{2 0}$ & $\mathbf{1 5}$ & $\mathbf{1 5 0}$ \\
\hline
\end{tabular}

ANOVA

\begin{tabular}{|l|l|l|l|l|l|l|}
\hline $\begin{array}{l}\text { Source } \\
\text { Variation }\end{array}$ & of & $d f$ & $M S$ & $F$ & P-value & F crit \\
\hline Rows & 28.31746 & 8 & 3.539683 & 10.83401 & $1.47 \mathrm{E}-08$ & 2.138229 \\
\hline Columns & 2.31746 & 6 & 0.386243 & 1.182186 & 0.331675 & 2.294601 \\
\hline Error & 15.68254 & 48 & 0.32672 & & & \\
\hline \multicolumn{7}{|l|}{} \\
\hline Total & 46.31746 & 62 & & & & \\
\hline
\end{tabular}

The ANOVA Two-way to find whether there is any significant impact of work life balance aspects and Work life balance of women at aviation sector

$\alpha=0.05$

Reject $\mathbf{H}_{0}$

Between Rows: 
F calculated value $=10.83401$ at $($ Degree of Freedom 8, 48)

Table Value: 2.138229

Since $\mathrm{F}$ cal value is $>$ than $\mathrm{F}$ table value

Reject $\mathrm{H}_{0}$

Between Columns:

F calculated value $=1.182186$ at (Degree of Freedom 6, 48)

Table Value 2.294601

Since F cal Value < Table Value

Accept $\mathrm{H}_{0}$

Hence, null hypothesis has been failed to be accepted, as such the results indicate that there is a significant impact of work life balance aspects on work life balance of women at aviation sector

Table 8: Crosstab of Work life balance at aviation sector and work life balance aspects

\begin{tabular}{|l|l|l|l|l|l|l|l|l|l|}
\hline $\begin{array}{l}\text { Age/workli } \\
\text { fe balnce } \\
\text { aspects }\end{array}$ & $\begin{array}{l}\text { Com } \\
\text { mitm } \\
\text { ent }\end{array}$ & $\begin{array}{l}\text { Adequate } \\
\text { and fair } \\
\text { compensati } \\
\text { on }\end{array}$ & $\begin{array}{l}\text { Job } \\
\text { satisfa } \\
\text { ction }\end{array}$ & $\begin{array}{l}\text { Trainin and } \\
\text { develop } \\
\text { ment }\end{array}$ & $\begin{array}{l}\text { Safe } \\
\text { and } \\
\text { health } \\
\text { y } \\
\text { worki } \\
\text { ng } \\
\text { condit } \\
\text { ions }\end{array}$ & $\begin{array}{l}\text { Skill } \\
\text { utiliz } \\
\text { ation } \\
\text { and } \\
\text { growt } \\
\text { h }\end{array}$ & $\begin{array}{l}\text { Socia } \\
\text { Integ } \\
\text { ratio } \\
\text { n }\end{array}$ & $\begin{array}{l}\text { Wor } \\
\text { k } \\
\text { envir } \\
\text { onm } \\
\text { ent }\end{array}$ & Total \\
\hline $\mathbf{1 7 - 2 5 y e a r s ~}$ & $\mathbf{1 5}$ & $\mathbf{1 0}$ & $\mathbf{1 8}$ & $\mathbf{1 5}$ & $\mathbf{1 3}$ & $\mathbf{1 2}$ & $\mathbf{1 6}$ & $\mathbf{1 2}$ & \\
\hline $\mathbf{2 5 - 3 5}$ & $\mathbf{7}$ & $\mathbf{5}$ & $\mathbf{7}$ & $\mathbf{4}$ & $\mathbf{8}$ & $\mathbf{7}$ & $\mathbf{4}$ & $\mathbf{3}$ & $\mathbf{3 2}$ \\
\hline Total & $\mathbf{2 2}$ & $\mathbf{1 5}$ & $\mathbf{2 5}$ & $\mathbf{1 9}$ & $\mathbf{2 1}$ & $\mathbf{1 9}$ & $\mathbf{2 0}$ & $\mathbf{1 5}$ & $\mathbf{1 5 0}$ \\
\hline
\end{tabular}

ANOVA

\begin{tabular}{|l|l|l|l|l|l|l|}
\hline $\begin{array}{l}\text { Source } \\
\text { Variation }\end{array}$ & of & $d f$ & $M S$ & $F$ & $P$-value & F crit \\
\hline Rows & 272.25 & 1 & 272.25 & 61.97561 & 0.000101 & 5.591448 \\
\hline Columns & 40 & 7 & 5.714286 & 1.300813 & 0.36871 & 3.787044 \\
\hline Error & 30.75 & 7 & 4.392857 & & & \\
\hline \multicolumn{7}{|l|}{} \\
\hline Total & 343 & 15 & & & & \\
\hline
\end{tabular}

2. The ANOVA to find out the impact of Age of respondents on work life balance aspects

$\alpha=0.05$

Reject $\mathrm{H}_{0}$ 
Between Rows:

F calculated value $=61.97561$ at $($ Degree of Freedom 1, 7)

Table Value: 5.591448

Since $\mathrm{F}$ cal value is $>$ than $\mathrm{F}$ table value

Reject $\mathrm{H}_{0}$

Between Columns:

F calculated value $=1.300813$ at $($ Degree of Freedom 7, 7)

Table Value 3.787044

Since F cal Value < Table Value

Accept $\mathrm{H}_{0}$

Hence, null hypothesis has been failed to be accepted, as such the results indicate that there is a significant impact of Age of respondents on work life balance aspects

\section{Conclusions}

1. It is concluded that most of the sample respondents (airhostess) of aviation sector are teenagers and youth.

2. It is clear from the sample respondents of airhostess majority are unmarried and chose to select airhostess profession as their choice of career.

3. Childcare, time stress, long working hours shift system are some of the

4. With regards to qualities of airhostess majority of respondents gave priority to style and glamorous, followed by multitasking, empathy and learning potential and kindness

5. With regards to Work life balance at aviation sector most of the respondents mentioned that it provides them multi cultural working environment, continuous progress and training, greater experience to work in aviation sector.

6. Some of the respondents felt fatigue and long working hours, such areas have to be looked at to minimize such aspects in order to balance work and life and gain greater support from organizational perspective.

7. Coming to the point of work life balance aspects job satisfaction is more among the airhostess, their commitment level, safe and healthy working conditions, and skill utilization and growth are positive aspects if aviation sector creates a healthy working culture, greater training and development with adequate and fair compensation, this sector would be very attractive for the airhostess to work for and make a sustainable work life balance.

8. Null hypothesis has been failed to be accepted, as such the results indicate that there is a significant impact of work life balance aspects on work life balance of women at aviation sector

Null hypothesis has been failed to be accepted, as such the results indicate that there is a significant impact of Age of respondents on work life balance aspects 


\section{References}

[1] Barnett, R. (1999). A New Work-Life Model for the Twenty-First Century. Annals of the American Academy of Political and Social Science, 56(2), 143-158.

[2] R.lockwood, N. (2003). Work life balance; challenges and solutions. 1-12

[3] Liang, S., \& Hsieh, A. (2007). Burnout and workplace deviance among flight attendants in Taiwan. Psychological Reports, 101(202), 457-468.

[4] Chung, C., \& Chung, U. (2009). An exploration of quality of life and related factors amongst female flight attendants. Journal of Nursing Research, 17, 212-219.

[5] Karasek, R. A., \& Theorell, T. (1990). Health work. Basic Book, New York.

[6] Brown, N. C., Prashantham, B. J., \& Abbott, M. (2003). Personality, social support and burnout among human service professionals in India. Journal of Community \& Applied Social Psychology, 13(4), 320-324.

[7] Fleetwood, S. (2007). Why work-life balance now?. The international journal of human resource management, 18(3), 387-400.

[8] Yadav, R. K., \& Dabhade, N. (2014). Work life balance and job satisfaction among the working women of banking and education sector-A comparative study. International Letters of Social and Humanistic Sciences, 21, 181-201.

[9] Williams, C. (2003). Sky service: The demands of emotional labour in the airline industry. Gender, Work \& Organization, 10(5), 513-550.

[10] Maeran, R., Pitarelli, F., \& Cangiano, F. (2013). Work-life balance and job satisfaction among teachers. Interdisciplinary Journal of Family Studies, 18(1).

[11] Beauregard, T. A., \& Henry, L. C. (2009). Making the link between work-life balance practices and organizational performance. Human resource management review, 19(1), 9-22.

[12] Barry, K. (2007). Femininity in flight: A history of flight attendants. Duke University Press.

[13] Ahmad, M. B., Wasay, E., \& Jhandir, S. U. (2012). Impact of employee motivation on customer satisfaction: Study of airline industry in Pakistan. Interdisciplinary Journal of Contemporary Research in Business, 4(6), 531-539.

[14] Eikhof, D. R. (2007). Introduction: what work? What life? What balance? Employee relations.

[15] Fan, W., Lam, J., Moen, P., Kelly, E., King, R., \& McHale, S. (2015). Constrained choices? Linking employees' and spouses' work time to health behaviors. Social Science \& Medicine, 126, 99-109. 